Sección Básica / Basic

Ensayo / Essay

\title{
Como lidiar con las desventajas de vivir en sociedades densas: reglas de tránsito en hormigas cortadoras de hojas
}

\author{
How to deal with the disadvantages of living in dense societies: \\ traffic rules in leaf-cutting ants
}

(D) ALEJANDRO G. FARJI-BRENER ${ }^{1}$

${ }^{1}$ Universidad Nacional del Comahue. Bariloche, Argentina, alefarji@yahoo.com

\begin{abstract}
*Autor para correspondencia
Alejandro G. Farji-Brener. Universidad Nacional del Comahue. Centro Universitario Bariloche, Laboratorio de Investigaciones en Hormigas (LIHO) - INIBIOMA-CONICET, Pasaje Gutiérrez 1125, (8400) Bariloche, Argentina, alefarji@yahoo.com

\section{Citación sugerida}

FARJI-BRENER, A. G. 2021. Como lidiar con las desventajas de vivir en sociedades densas: reglas de tránsito en hormigas cortadoras de hojas. Revista Colombiana de Entomología 47 (2): e11046. https://doi.org/10.25100/socolen. v47i2.11046

Recibido: 02-Mar-2021

Aceptado: 07-Jul-2021

Publicado: 26-Oct-2021

Revista Colombiana de Entomología ISSN (Print): 0120-0488

ISSN (On Line): 2665-4385

https://revistacolombianaentomologia.univalle.edu.co

Open access

(c) (i) (2) (2) BY-NC-SA 4.0
\end{abstract}

Publishers: Sociedad Colombiana de Entomología SOCOLEN (Bogotá, D. C., Colombia) https://www.socolen.org.co

Universidad del Valle (Cali, Colombia)

https://www.univalle.edu.co

(C) 2021 Sociedad Colombiana de Entomología SOCOLEN y Universidad del Valle - Univalle
Resumen: Transportar alimentos desde los centros de producción hacia los centros de consumo es un desafío para las sociedades densamente pobladas. Para evitar retrasos, el sistema de senderos debe ser eficiente y el comportamiento de los transportistas debe reducir la probabilidad de colisiones. En este trabajo se describe cómo las hormigas cortadoras de hojas $(\mathrm{HCH})$ lidian con ese dilema, a través de la construcción de senderos de forrajeo que minimizan los embotellamientos y mediante comportamientos que reducen los retrasos. Por un lado, las $\mathrm{HCH}$ construyen senderos que son suficientemente anchos como para evitar aglomeraciones en los momentos de máxima actividad. En adición, en lo puntos de bifurcación la suma del ancho de las ramificaciones siempre es mayor que el sendero troncal precedente, lo cual agiliza el tránsito. Finalmente, las HCH construyen bifurcaciones del sendero troncal con ángulos que reducen el costo de mantenimiento del nuevo tramo o del recorrido total, dependiendo de cuál factor sea más limitante. Por otra parte, las hormigas muestran un abanico de comportamientos que evitan los retrasos: pueden viajar sobre los fragmentos de hojas y reducir así la densidad de flujo, remueven obstáculos para minimizar aglomeraciones, acarrean cargas pesadas en los momentos de menor tránsito, mantienen el carril al doblar, y establecen prioridades en situaciones de "embotellamientos". Estos ejemplos ilustran cómo las hormigas, emplean reglas simples de comportamiento que surgen de interacciones positivas entre los individuos, para resolver problemas complejos como la regulación del tránsito.

Palabras clave: Acromyrmex, Atta, auto-organización, decisiones colectivas, fenotipo extendido, forrajeo, reglas de tránsito, pistas de forrajeo.

Abstract: Transporting food from production centers to consumption centers is a challenge for densely populated societies. To avoid delays, the trail system must be efficient and the behavior of the transporters must reduce the probability of collisions. This work, describes how Leaf-Cutting Ants (LCA) solve these dilemmas by optimizing their trail design and performing behaviors that avoid bottlenecks and delays. On the one hand, the LCA build trails wide enough to avoid traffic jams at times of peak foraging activity. Also, at the bifurcation points, the sum of the branching trail widths is always higher than the width of the precedent trunk trail. Finally, LCA builds their branching trails with angles that reduce the maintenance cost of the new trail sector or of the total trail length, depending on which factor is more limiting. On the other hand, LCA shows a range of behaviors that avoid delays: minima workers can travel on the leaf fragments thereby reducing flux density, unloaded workers remove obstacles from the trail, ants carry extra-large loads mainly during situations of low traffic, ants maintain their lane when turning, and foragers show priority rules during jam situations. These examples illustrate how ants, using simple behavioral rules that arise from positive interactions among individuals, can solve complex problems such as traffic regulation.

Keywords: Atta, Acromyrmex, collective decisions, extended phenotype, foraging, self-organization, traffic rules

\section{Introducción}

Los problemas de tránsito al vivir en sociedades densas. Uno de los grandes problemas de las sociedades densas es transportar el alimento desde los centros de producción hacia los centros de consumo. Dado el alto volumen de recursos que se debe trasladar, este proceso presenta dos grandes desafíos que hay que resolver para que sea eficiente. Por un lado, requiere de diseños de senderos que eviten aglomeraciones. Y, por otra parte, precisa de comportamientos de los transportistas que reduzcan la probabilidad de colisiones y retrasos (Burd et al. 2002). En consecuencia, para reducir el costo de demoras en el transporte de alimento, las sociedades altamente pobladas deben diseñar, construir y mantener un eficiente sistema de senderos, y los 
individuos que trasladan el alimento deben presentar comportamientos que reduzcan demoras.

Las hormigas cortadoras de hojas $(\mathrm{HCH})$ son un modelo ideal para estudiar cómo las sociedades densas optimizan el transporte de alimento (Burd 2006; Forucasié et al. 2010). En primer lugar, estas hormigas forman sociedades que se componen desde cientos de miles (género Acromyrmex) y hasta millones de individuos (género Atta), por lo cual requieren movilizar una enorme cantidad de alimento para mantener la colonia (Hölldobler y Wilson 2010). Segundo, las HCH presentan un sistema de senderos que posee alto tránsito, lo cual incrementa la probabilidad de colisiones y retrasos. Estos insectos cortan hojas de diversas plantas, cuyos fragmentos son transportados hacia el nido para cultivar un hongo simbionte, alimento principal de las larvas (Hölldobler y Wilson 2010). Dado que forrajean en forma simultánea sobre diferentes plantas alrededor de su nido, presentan un sistema de caminos de forma dendrítica que sale del hormiguero y se bifurca hacia los diferentes parches de vegetación (Fig. 1). Por allí transitan hormigas cargadas que regresan al nido y hormigas sin carga que van en búsqueda de hojas, lo que provoca eventuales colisiones entre obreras que van en direcciones contrarias. Por otra parte, las hormigas cargadas pueden diferir en el tamaño de la carga que llevan, caminando más despacio y provocando retrasos en sus compañeras. Todo esto implica que se requiere un diseño eficiente de senderos para evitar aglomeraciones y demoras en el ingreso de alimento al nido (Burd et al. 2002; Forucasié et al. 2010). Tercero, las HCH son insectos comunes y fáciles de detectar en las regiones que habitan. Finalmente, tanto las hormigas, como las características de sus senderos de forrajeo son factibles de manipular en campo o en laboratorio. Todos estos rasgos hacen de las $\mathrm{HCH}$ un organismo ideal para estudiar cómo las sociedades densas logran reducir el problema de transportar alimento a través de largas distancias.

En este trabajo se describe los principales resultados de las últimas investigaciones sobre cómo las $\mathrm{HCH}$ optimizan la eficiencia en la construcción de su sistema de senderos y en el transporte de alimento. Para ello, primero se describen las características de sus senderos que incrementan su eficiencia, y segundo, se detallan los comportamientos que reducen la probabilidad de colisiones y retrasos en el flujo de alimento hacia el nido.

Las hormigas cortadoras como "ingenieras" de caminos. Dado que la selección natural puede actuar sobre los individuos a través de sus construcciones, se propone que éstas son extensiones de su fenotipo (Dawkins 1982). Construcciones ineficientes implican un gasto de energía que podrían derivarse a otras funciones esenciales y pueden tener consecuencias
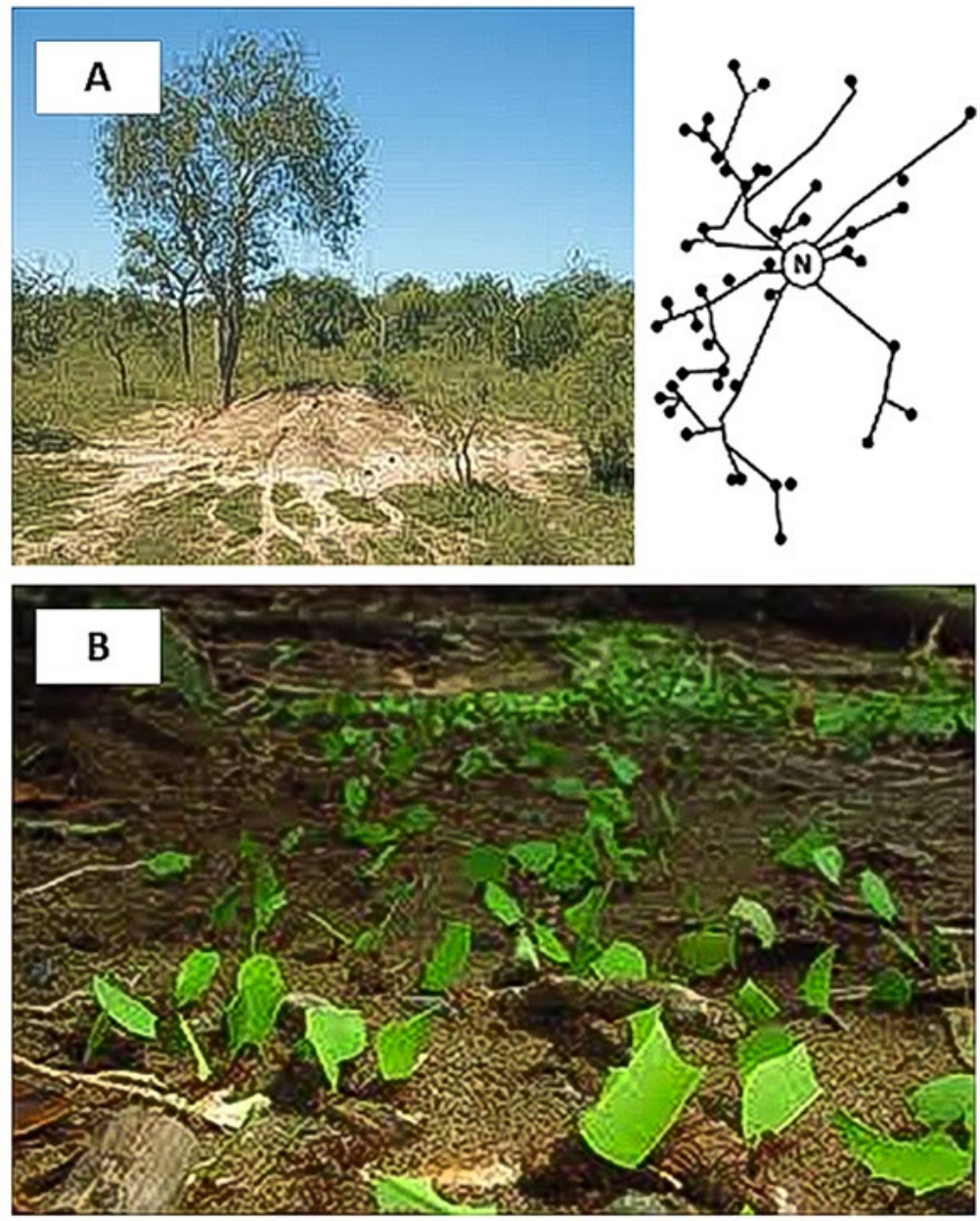

Figura 1. Esquema del sistema de senderos $(\mathrm{N}=\mathrm{Nido})$ de hormigas cortadoras de hojas (A, izquierda) y foto de un nido de Atta wollenveideri en pastizales subtropicales de Argentina, donde se puede apreciar la naturaleza dendrítica de los caminos por donde normalmente circula una alta densidad de hormigas acarreando hojas (B). 
negativas sobre la adecuación de los organismos como reducir la captura de alimento o proveer refugios inadecuados (Turner 2000). En consecuencia, se espera que la selección natural penalice los comportamientos que generan construcciones ineficientes y favorezca las conductas que generan construcciones más eficientes. Los senderos de forrajeo de las $\mathrm{HCH}$ son construcciones que representan un claro ejemplo de fenotipos extendidos (Farji-Brener et al. 2015). Por estos senderos, que las propias hormigas mantienen libres de hojarasca, transitan las obreras que salen del nido en búsqueda de alimento y las que regresan cargadas con fragmentos de hojas hacia el nido (Silva et al. 2013). Estos senderos poseen varias ventajas; proveen una señal física de reclutamiento, facilitan la deposición de feromonas, promueven el intercambio de información, e incrementan la velocidad del transporte (Dossutour et al. 2007, Farji-Brener et al. 2010a). Sin embargo, para que estas funciones se realicen de forma exitosa, su diseño debe ser eficiente. En este sentido, la construcción de los senderos de forrajeo representa para las hormigas un problema similar al cual se enfrentan los ingenieros de caminos; su diseño debe optimizarse aumentando beneficios y reduciendo costos. Esta optimización se logra si la forma de los senderos no genera retrasos y embotellamientos en los momentos de máximo flujo (Burd et al. 2002; Burd 2006). Por ejemplo, el ancho de los senderos debe permitir un tránsito fluido en los momentos de máxima actividad. Esto ocurre en Atta cephalotes y Atta colombica, en donde el ancho de los senderos principales permite un tránsito fluido cuando la cantidad de hormigas que forrajean es máxima (Burd et al. 2002). Más aún, en lo puntos de bifurcación la suma del ancho de las ramificaciones siempre es mayor que el sendero troncal precedente (Fig. 2). De esta manera, las hormigas que regresan cargadas por las bifurcaciones pueden moverse libremente por el carril central del sendero, en donde hay menos obstáculos de la vegetación circundante, reduciendo la probabilidad de embotellamientos y eventuales retrasos del ingreso de alimento al nido (Farji-Brener et al. 2012). Finalmente, muchas veces las $\mathrm{HCH}$ integran ramas caídas o raíces expuestas como parte de sus caminos, reduciendo los costos de construcción y mantenimiento e incrementando así la velocidad de transporte (Farji-Brener et al. 2007).
Por otra parte, cuando se construye una bifurcación que conecta el sendero troncal con un nuevo recurso, el ángulo de esta bifurcación puede reducir el costo de mantenimiento del nuevo tramo o el tiempo de viaje total, pero no ambas cosas simultáneamente. Por ejemplo, construir una nueva bifurcación lo más corta posible (por ejemplo, a $90^{\circ}$ del sendero troncal), implica menor mantenimiento (por ejemplo menor área del nuevo tramo para mantener), pero también un viaje total más largo; y construir la nueva bifurcación a un ángulo de $45^{\circ}$ del sendero troncal implica mayor mantenimiento -dado que el nuevo tramo es más largo-, pero menor tiempo de transporte, por ser el recorrido total más corto (Fig. 3). Un trabajo reciente encontró que los nidos ubicados en bosques cerrados, en donde la caída constante de hojarasca implica un alto costo de mantenimiento, pero donde el dosel protege a las hormigas del sol y la lluvia reduciendo el costo de viaje, las bifurcaciones poseen ángulos cercanos a los $90^{\circ}$. Pero los nidos ubicados en ambientes abiertos, en donde el costo de mantenimiento es menor al no haber caída de hojarasca, aunque el costo de traslado sea mayor por estar expuestos directamente al sol y la lluvia, poseen bifurcaciones con ángulos cercanos a $\operatorname{los} 45^{\circ}$ (Farji-Brener et al. 2015, Fig. 3). En resumen, estos ejemplos demuestran cómo los senderos de las $\mathrm{HCH}$ presentan características que optimizan el flujo y reducen los costos que implicarían un retraso de ingreso de alimento al nido.

Las reglas del buen transportista. De nada vale tener senderos diseñados para reducir retrasos, si los individuos que transportan los alimentos realizan maniobras peligrosas que generan colisiones o poseen comportamientos que retrasan al resto de los transportistas. Diversos estudios demuestran cómo las $\mathrm{HCH}$ poseen comportamientos que reducen los choques y las demoras en los senderos. Por ejemplo, las hormigas sin carga remueven obstáculos en el sendero (Alma et al. 2020), inclusive aquellos obstáculos a alturas que no representan un problema para ellas, pero lo serán para las hormigas cargadas que regresan al nido (Bruce et al. 2017).

Una reducción experimental en el ancho de los senderos de hormigas del género Acromyrmex incrementó el número de obreras de tamaño pequeño que viajaban sobre los fragmentos de hojas transportados por las obreras mayores. Esto
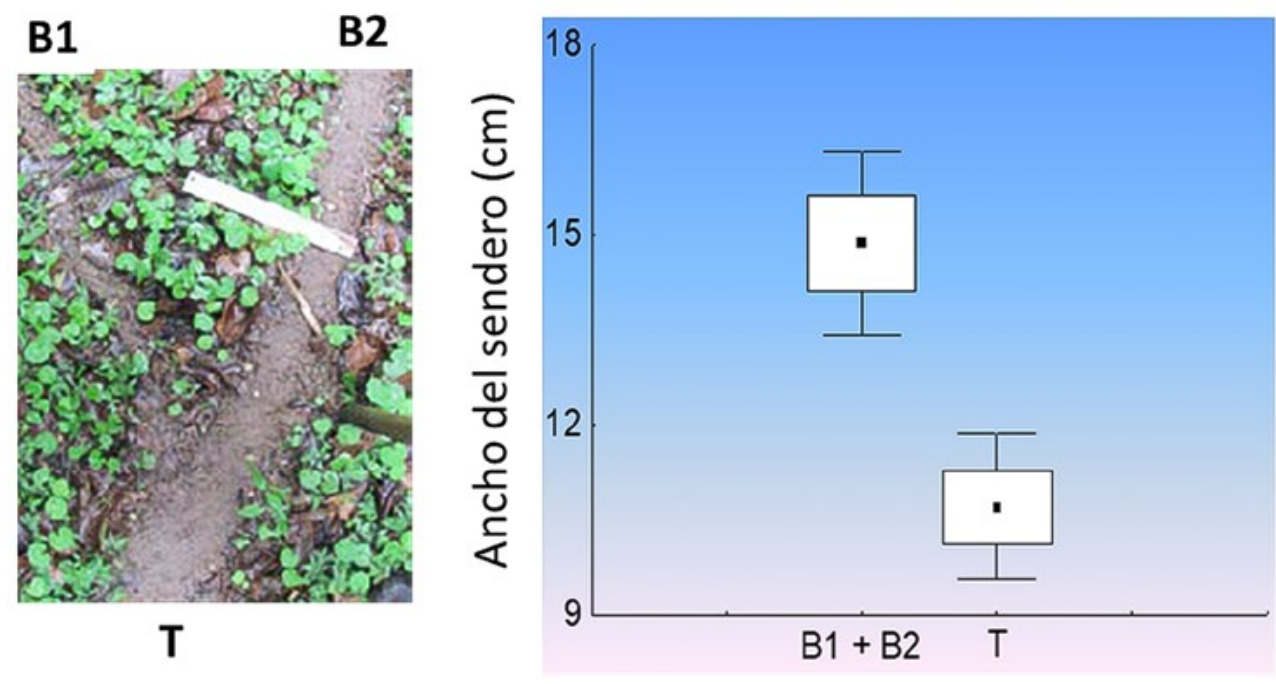

Figura 2. Ejemplo de cómo la suma del ancho de las bifurcaciones (B1 y B2) es mayor que el ancho del sendero troncal precedente $(\mathrm{T})$. De esta manera, se reduce la probabilidad de embotellamientos en los momentos de máximo flujo (Farji-Brener et al. 2012). 




Figura 3. Al crear una nueva bifurcación para unir un sendero troncal con un recurso recién descubierto, se la puede construir con un ángulo cercano a $90^{\circ}$ reduciendo así el mantenimiento del nuevo sector, pero incrementando el tiempo total de viaje desde el recurso al nido (opción 1); o con un ángulo menor, incrementando el costo de mantenimiento del nuevo sector (al ser más largo), pero reduciendo el costo de viaje por ser el recorrido total más corto (opción 2). Nidos ubicados en bosques cerrados -donde la caída de hojarasca implica un alto costo de mantenimiento, pero el dosel protege del sol y la lluvia reduciendo el costo de viaje-, construyen bifurcaciones más cercanas a $90^{\circ}$; pero nidos en ambientes abiertos -en donde el costo de mantenimiento es menor al no caer hojarasca, pero el costo de traslado es mayor por estar expuestos directamente al sol y la lluvia-, construyen bifurcaciones con ángulos cercanos a los $45^{\circ}$ (Farji-Brener et al. 2015).

sugiere que este comportamiento de las obreras mínimas ("hitchhiker", en inglés) puede haber sido seleccionado no solo para defender a las obreras de los parasitoides sino también para reducir las aglomeraciones, dado que al "viajar" sobre las hojas reducen la densidad de hormigas en el sendero (Hastenreiter et al. 2018). Además, en senderos muy angostos se observó un comportamiento de segregación temporal en el movimiento de $\mathrm{HCH}$ cargadas y sin carga, sugiriendo la existencia de reglas de prioridad en el paso (Dussutour et al. 2009).

Por otra parte, observaciones en especies de Atta y Acromyrmex confirmaron que las hormigas mantienen su carril al doblar (por ejemplo, al ingresar al sendero troncal desde una bifurcación), reduciendo la probabilidad de colisiones y retrasos (Cibils-Martina et al. 2017, Fig. 4). Finalmente, existen hormigas que llevan cargas extra-grandes por lo cual caminan lento y retrasan al resto que van detrás, al igual que los camiones pesados retrasan al resto de vehículos en una autopista. Trabajos recientes reportan que este comportamiento es inusual a grandes flujos, pero común cuando la actividad de forrajeo es baja, sugiriendo la existencia de "horarios restringidos" para cargas pesadas, como ocurre en las sociedades humanas (Farji-Brener et al. 2010b). Un estudio experimental confirmó esta hipótesis; las hormigas preferían acarrear cebos más grandes principalmente en condiciones de bajo flujo (Pereyra y Farji-Brener 2020, Fig. 5). En resumen, las HCH muestran diversos comportamientos que reducen las aglomeraciones como la remoción "altruista" de obstáculos, el viajar sobre la carga, la segregación temporal, la existencia de reglas de prioridades, el mantenimiento del carril al doblar, y la restricción de transportar cargas grandes en momentos de alto flujo.
Dime cómo construyes y te diré cómo eres: ventajas de estudiar fenotipos extendidos en artrópodos. En este trabajo se resume cómo las $\mathrm{HCH}$ resuelven conflictos de tránsito a través de sus comportamientos (Tabla 1). Parte de estos comportamientos se expresan en el diseño de sus senderos, los cuales son considerados extensiones de su fenotipo (Farji-Brener et al. 2015). Como fue discutido, la adecuación de un organismo se puede estudiar analizando directamente sus conductas o estudiando sus construcciones (Turner 2000). Esta última aproximación, aunque menos explorada, posee varias ventajas. Primero, las construcciones que realizan los organismos muchas veces son más sencillas de encontrar y manipular que los propios individuos. Segundo, no siempre es necesaria la presencia de los organismos para estudiar sus construcciones, lo cual amplia las condiciones en las cuales puede realizarse una investigación. No es necesaria la presencia de hormigas para estudiar sus senderos, ni de las termitas para estudiar sus termiteros, ni de las arañas para estudiar sus telas, ni de los galígenos para estudiar las agallas, ni de las larvas de neurópteros para estudiar sus trampas (Stone et al. 1998; Nakata 2012; Farji-Brener et al. 2008; Zachariah et al. 2020).

Finalmente, las construcciones pueden reflejar cómo los organismos resuelven situaciones que requieren una solución de compromiso ("trade-off", en inglés), ofreciendo una oportunidad única para comprender la adecuación de los comportamientos en situaciones conflictivas. Por ejemplo, las larvas de ciertos neurópteros ("antlions", en inglés) construyen en el suelo trampas cónicas de caída con las cuales capturan insectos caminadores, principalmente hormigas. Pero al construir las trampas cónicas deben elegir entre incrementar 


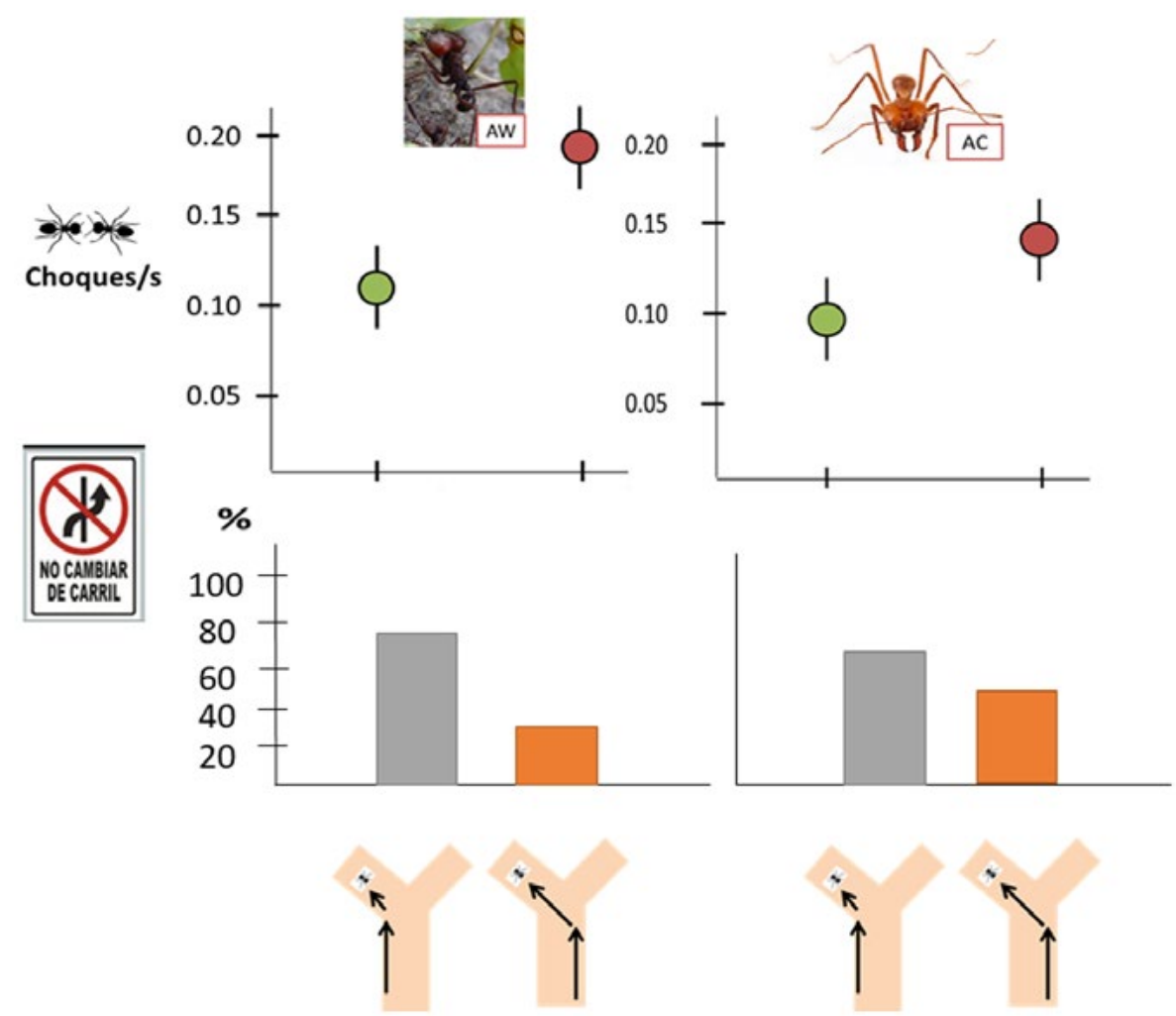

Figura 4. Las hormigas de Atta wollenvenderi y Atta cephalotes normalmente mantienen su carril al ingresar al sendero troncal desde una bifurcación (abajo), lo cual reduce el número de choques entre hormigas (arriba, color verde) incrementando así la tasa de entrada de alimento al nido (Cibilis-Martina et al. 2017).

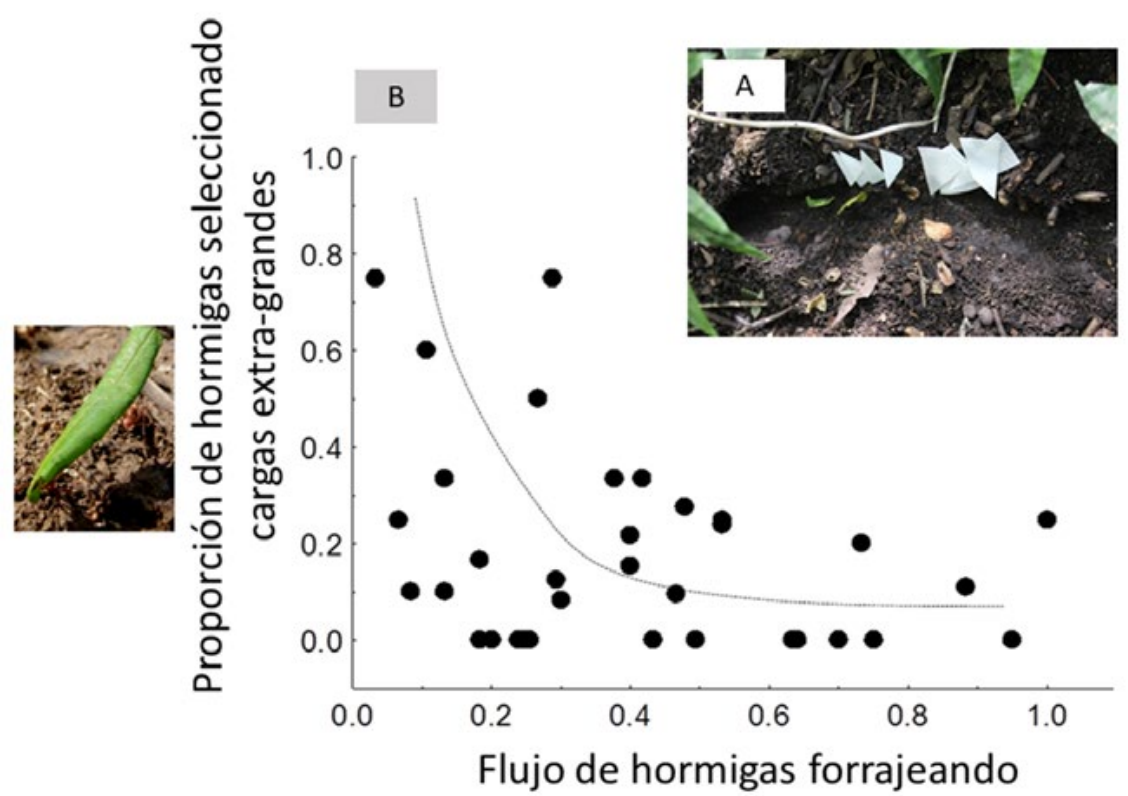

Figura 5. Resultado de un experimento en donde se ofrecen cebos (papeles embebidos en jugo de naranja) de tamaño extra-grande y mediano (A). Las hormigas seleccionaban acarrear los fragmentos extra-grandes principalmente cuando había poco flujo de obreras en el camino (B), reduciendo la probabilidad de generar retrasos en sus compañeras que van detrás (Farji-Brener et al 2010b, Pereyra y Farji-Brener 2020). 
su diámetro para aumentar la probabilidad de captura, o construir paredes con mayor pendiente para evitar que las presas se escapen, ya que ambas características de la trampa no se pueden optimizar simultáneamente (Lomáscolo y Farji-Brener 2001; Farji-Brener y Amador-Vargas 2020). Las arañas que construyen telas orbiculares deben elegir entre incrementar la probabilidad de intercepción de presas aumentando el diámetro de su tela pegajosa, o aumentar la probabilidad de retención construyendo un entramado más denso, ya que es difícil aumentar ambas cosas al mismo tiempo (Blackledge y Eliason 2007). Finalmente, si las hormigas cortadoras de hojas incrementan el ancho de la entrada de sus nidos reducen la probabilidad de aglomeraciones, pero incrementan la probabilidad de inundaciones (Sendoya et al. 2014; Rodríguez-Planes y Farji-Brener 2019). En resumen, estudiar el comportamiento de los artrópodos a través de sus construcciones no solo puede ser más sencillo, sino que también nos puede permitir comprender mejor cómo los organismos enfrentan problemas cuyas resoluciones son excluyentes.

Tabla 1. Resumen de reglas de tránsito en hormigas cortadoras de hojas.

\begin{tabular}{|c|c|c|c|}
\hline Tipo de regla & Característica & Consecuencia & Ejemplo ilustrado \\
\hline Construcción & Senderos troncales anchos & $\begin{array}{l}\text { Reducción de aglomeraciones } \\
\text { en los momentos de máxima } \\
\text { actividad de forrajeo }\end{array}$ & \\
\hline Construcción & $\begin{array}{l}\text { Bifurcaciones cuyos anchos } \\
\text { sumados son mayores que el } \\
\text { sendero troncal }\end{array}$ & $\begin{array}{l}\text { Reducción de aglomeraciones } \\
\text { en los momentos de máxima } \\
\text { actividad de forrajeo }\end{array}$ & \\
\hline Construcción & $\begin{array}{l}\text { Plasticidad en los ángulos de las } \\
\text { bifurcaciones }\end{array}$ & $\begin{array}{l}\text { Reducción del costo de mante- } \\
\text { nimiento del nuevo sector o del } \\
\text { viaje total, dependiendo de cuál } \\
\text { factor es más limitante }\end{array}$ & \\
\hline Construcción & $\begin{array}{l}\text { Uso de ramas caídas como } \\
\text { senderos }\end{array}$ & $\begin{array}{l}\text { Reducción de costos de cons- } \\
\text { trucción y mantenimiento, } \\
\text { incremento en la velocidad de } \\
\text { transporte }\end{array}$ & \\
\hline Comportamiento & $\begin{array}{l}\text { Remoción de obstáculos en el } \\
\text { sendero }\end{array}$ & $\begin{array}{l}\text { Reducción de aglomeraciones } \\
\text { en los momentos de máxima } \\
\text { actividad de forrajero }\end{array}$ & \\
\hline Comportamiento & $\begin{array}{l}\text { Obreras mínimas viajan sobre } \\
\text { las hojas acarreadas por otras } \\
\text { obreras }\end{array}$ & $\begin{array}{l}\text { Reducción en la densidad de } \\
\text { tránsito }\end{array}$ & \\
\hline Comportamiento & Reglas de prioridad de paso & $\begin{array}{l}\text { Reducción de las congestiones } \\
\text { en momentos de alto flujo }\end{array}$ & \\
\hline Comportamiento & Mantener el carril al doblar & $\begin{array}{l}\text { Reducción de la probabilidad de } \\
\text { colisiones y retrasos }\end{array}$ & \\
\hline Comportamiento & $\begin{array}{l}\text { Llevar cargas extra-grandes } \\
\text { principalmente en momentos de } \\
\text { bajo flujo }\end{array}$ & $\begin{array}{l}\text { Reducción de la probabilidad de } \\
\text { generar retrasos en las forrajeras } \\
\text { que van detrás }\end{array}$ & $\infty$ \\
\hline
\end{tabular}


Conclusiones finales: cómo lograr un tránsito fluido sin la necesidad de policías o infracciones. Trabajos relativamente recientes han empleado a las hormigas para comprender o mejorar las reglas de tránsito vehicular o peatonal en humanos (Peters et al. 2006; Claes y Holvoet 2012; Jabbarpour et al. 2014). Como se ha descrito, ciertas pautas en las colonias de hormigas cortadoras de hojas son muy similares a las que ocurren en las urbes humanas: el mantenimiento del carril al doblar, las restricciones horarias para transportes que llevan cargas pesadas y las prioridades de paso son reglas comunes en ambas sociedades. Pero pese a estas similitudes, el tránsito en hormigas y humanos presenta ciertas diferencias que generalmente complican las comparaciones y eventuales aplicaciones (Forucasié et al. 2010). Primero, todas las hormigas que circulan por los senderos de forrajeo pertenecen al mismo hormiguero y comparten el mismo objetivo: buscar y transportar alimento para la colonia. Sin embargo, vehículos o transeúntes que circulan por un mismo camino muchas veces poseen objetivos y destinos finales diferentes, reduciendo la probabilidad de cooperación. Esto explica el motivo por el cual el tránsito en humanos es regido por restricciones negativas externas (policías y multas) mientras que la regulación del tránsito en hormigas es regida por las interacciones positivas internas (por ejemplo, intercambio de información, reclutamiento). Segundo, las hormigas no poseen las mismas limitaciones físicas que los vehículos o transeúntes. Debido a su baja masa y poca inercia, los choques entre hormigas no les generan daño alguno. Más aún, estas colisiones muchas veces son aprovechadas para intercambiar información sobre la ubicación de una nueva fuente de alimento (Farji-Brener et al. 2010a). En consecuencia, es necesario cierta precaución al emplear a las hormigas como modelo para comprender o mejorar las reglas de tránsito en sociedades humanas.

En este trabajo se ha resumido cómo los diseños de los senderos y los comportamientos de los individuos pueden reducir conflictos de tránsito en $\mathrm{HCH}$ y así mitigar uno de los grandes problemas de vivir en sociedades densas: el transporte del alimento desde los centros de producción (por ejemplo, los parches de vegetación seleccionados) hacia los centros de consumo (por ejemplo, el hormiguero). Esto ilustra la manera como las hormigas pueden resolver conflictos complejos -como la regulación del tránsito-, usando reglas simples de comportamiento que no son impuestas de manera externa, sino que surgen de interacciones directas e indirectas entre los individuos.

\section{Literatura citada}

ALMA, A. M.; FARJI-BRENER, A. G.; ELIZALDE, L. 2020. With a little help from my friends: Individual and collaborative performance during trail clearing in leaf-cutting ants. Biotropica 52 (3): 554-562. https://doi.org/10.1111/btp.12770

BLACKLEDGE, T. A.; ELIASON, C. M. 2007. Functionally independent components

of prey capture are architecturally constrained in spider orb webs. Biology Letters 3 (5): 456-458. https://doi.org/10.1098/ rsbl.2007.0218

BRUCE, A. I.; CZACZKES, T. J.; BURD, M. 2017. Tall trails: ants resolve an asymmetry of information and capacity in collective maintenance of infrastructure. Animal Behavior 127: 179-185. https://doi.org/10.1016/j.anbehav.2017.03.018

BURD, M.; D. ARCHER; N. ARANWELA; STRADLING, D. J. 2002. Traffic dynamics of the leaf-cutting ant Atta cepha- lotes. The American Naturalist 159 (3): 283-293. https://doi. org/10.1086/338541

BURD, M. 2006. Ecological consequences of traffic organization in ant societies. Physica A: Statistical Mechanics and its Applications 372 (1): 124-131. https://doi.org/10.1016/j.physa.2006.05.004

CIBILS-MARTINA, L.; ELIZALDE, L.; FARJI-BRENER, A. G. 2017. Traffic rules around the corner: walking of leaf-cutting ants at branching points in trunk trails. Insectes Sociaux 64 (4): 549-555. https://doi.org/10.1007/s00040-017-0576-5

CLAES, R.; HOLVOET, T. 2012. Cooperative ant colony optimization in traffic route calculations. In Advances on Practical Applications of Agents and Multi-Agent Systems (pp. 23-34). Springer, Berlin, Heidelberg. https://doi.org/10.1007/978-3-64228786-2 3

DAWKINS, R. 1982. The extended phenotype (Vol. 8). Oxford University Press. New York, $440 \mathrm{p}$.

DOSSUTOUR, A.; BESHERS, S.; DENEUBOURG, J. L.; FOURCASSIÉ, V. 2007. Crowding increases foraging efficiency in the leaf-cutting ant Atta colombica. Insectes Sociaux 54 (2): 158165. https://doi.org/10.1007/s00040-007-0926-9

DUSSUTOUR, A.; BESHERS, S.; DENEUBOURG, J. L.; FOURCASSIE, V. 2009. Priority rules govern the organization of traffic on foraging trails under crowding conditions in the leaf-cutting ant Atta colombica. Journal of Experimental Biology 212 (4): 499-505. https://doi.org/10.1242/jeb.022988

FARJI-BRENER, A. G.; BARRANTES, G.; LAVERDE, O.; FIERRO-CALDERÓN, K.; BASCOPÉ, F.; LÓPEZ, A. 2007. Fallen branches as part of leaf-cutting ant trai1s: their role in resource discovery and leaf transport rates in Atta cephalotes. Biotropica 39 (2): 211-215. https://doi.org/10.1111/j.1744-7429.2006.00256.x

FARJI-BRENER, A.G.; CARVAJAL, D.; GEI, M. G.; OLANO, J.; SÁNCHEZ, J. D. 2008. Direct and indirect effects of soil structure on the density of an antlion larva in a tropical dry forest. Ecological Entomology 33 (2): 183-188. https://doi.org/10.1111/ j.1365-2311.2007.00948.x

FARJI-BRENER, A. G.; AMADOR-VARGAS, S.; CHINCHILLA, F.; ESCOBAR, S.; CABRERA, S.; HERRERA, M. I.; SANDOVAL, C.. 2010a. Information transfer in head-on encounters between leaf-cutting ant workers: food, trail condition or orientation cues? Animal Behavior 79 (2): 343-349. https://doi. org/10.1016/j.anbehav.2009.11.009

FARJI-BRENER, A. G.; CHINCHILLA, F. A.; RIFKIN, S.; SÁNCHEZ CUERVO, A. M.; TRIANA, E; QUIROGA, V; GIRALDO, P. 2010b. The 'truck-driver' effect in leaf-cutting ants: how individual load influences the walking speed of nest-mates. Physiological Entomology 36 (2): 128-134. https://doi.org/10.1111/ j.1365-3032.2010.00771.x

FARJI-BRENER, A. G.; MORUETA-HOLME, N.; CHINCHILLA, F.; WILLINK, B.; OCAMPO, N.; BRUNER, G. 2012. Leaf-cutting ants as road engineers: the width of trails at branching points in Atta cephalotes. Insectes sociaux 59 (3): 389-394. https://doi. org/10.1007/s00040-012-0231-0

FARJI-BRENER, A. G.; CHINCHILLA, F.; UMAÑA, M. N.; OCASIO-TORRES, M. E.; CHAUTA-MELLIZO, A.; ACOSTA-ROJAS, D.; MARINARO, S.; DE TORRES CURTH, M.; AMADOR-VARGAS, S. 2015. Branching angles reflect a trade-off between reducing trail maintenance costs or travel distances in leaf-cutting ants. Ecology 96 (2): 510-517. https://doi. org/10.1890/14-0220.1

FARJI-BRENER, A. G.; AMADOR-VARGAS, S. 2020. Plasticity in extended phenotypes: how the antlion Myrmeleon crudelis adjusts the pit traps depending on biotic and abiotic conditions. Israel Journal of Ecology and Evolution, 66 (1-2): 41-47. https:// doi.org/10.1163/22244662-20191055

FORUCASIÉ V.; DUSSUTOUR A.; DENEUBOURG, J. L. 2010. Ant traffic rules. Journal of Experimental Biology 213 (14): 2357-2363. https://doi.org/10.1242/jeb.031237 
HASTENREITER, I. N.; LOPES, J. F. S.; DA SILVA CAMARGO, R.; FORTI, L. C. 2018. Avoiding traffic jams: Hitchhiking behavior as a strategy to reduce ant workers' traffic on the foraging trail. Behavioral processes 157: 54-58. https://doi.org/10.1016/j. beproc. 2018.08 .015

HÖLLDOBLER, B.; WILSON, E. O. 2010. The leafcutter ants: civilization by instinct. WW Norton \& Company. New York, London, $192 \mathrm{p}$.

JABBARPOUR, M. R.; MALAKOOTI, H.; NOOR, R. M.; ANUAR, N. B.; KHAMIS, N. 2014. Ant colony optimization for vehicle traffic systems: applications and challenges. International Journal of Bio-Inspired Computation 6 (1): 32-56. https:// doi.org/10.1504/IJBIC.2014.059970

NAKATA, K. 2012. Plasticity in an extended phenotype and reversed up-down asymmetry of spider orb webs. Animal Behavior 83 (3): 821-826. https://doi.org/10.1016/j.anbehav.2011.12.030

LOMÁSCOLO, S.; FARJI-BRENER, A. G. 2001. Adaptive shortterm changes in pit design by antlion larvae (Myrmeleon sp.) in response to different prey conditions. Ethology, Ecology and Evolution 13 (4): 393-397. https://doi.org/10.1080/08927014.2 001.9522770

PEREYRA, M.; FARJI-BRENER, A. G. 2020. Traffic restrictions for heavy vehicles: Leaf-cutting ants avoid extra-large loads when the foraging flow is high. Behavioral Processes 170: 104014. https://doi.org/10.1016/j.beproc.2019.104014

PETERS, K.; JOHANSSON, A.; DUSSUTOUR, A.; HELBING, D. 2006. Analytical and numerical investigation of ant behavior under crowded conditions. Advances in Complex Systems 9 (4): 337-352. https://doi.org/10.1142/S0219525906000859

RODRÍGUEZ-PLANES, L. I.; FARJI-BRENER, A. G. 2019. Extended phenotypes and foraging restrictions: ant nest entrances and resource ingress in leaf-cutting ants. Biotropica 51 (2): 178-185. https://doi.org/10.1111/btp.12630
SENDOYA, S.; SILVA, P. D.; FARJI-BRENER, A. G. 2014. Does inundation risk affect leaf-cutting ant distribution? A study along a topographic gradient of a Costa Rican tropical wet forest. Journal of Tropical Ecology 30 (1): 89-92. https://doi.org/10.1017/ S026646741300076X

SILVA, P.; BIEBER, A. G. D.; KNOCH, T. A.; TABARELLI, M.; LEAL, I. R.; WIRTH, R. 2013. Foraging in highly dynamic environments: Leaf-cutting ants adjust foraging trail networks to pioneer plant availability. Entomologia Experimentalis et Applicata 147 (2): 110-119. https://doi.org/10.1111/eea.12050

STONE, G. N.; COOK, J. M. 1998. The structure of cynipid oak galls: patterns in the evolution of an extended phenotype. Proceedings of the Royal Society of London. Series B: Biological Sciences 265 (1400): 979-988. https://doi.org/10.1098/ rspb.1998.0387

TURNER, J. S. 2000. The extended organism: the physiology of animal-built structures. Harvard University Press. Cambrige, UK, $256 \mathrm{p}$.

ZACHARIAH, N.; SINGH, S.; MURTHY, T. G.; BORGES, R. M. 2020. Bi-layered architecture facilitates high strength and ventilation in nest mounds of fungus-farming termites. Scientific Reports 10 (1): 131157. https://doi.org/10.1038/s41598-02070058-2

\section{Contribuciones de los autores}

Alejandro G. Farji-Brener: conceptualización, curaduría de datos, escritura (borrador original), escritura (correcciones del arbitraje y de edición).

\section{Conflictos de interés}

El autor declara no tener conflictos de intereses. 